\title{
Atypical Atrial Flutter in a Patient with Ischemic Stroke - A Rare ECG Finding
}

\author{
Authors \\ Dr Surender Kumar ${ }^{1}$, Dr Hitender Kumar ${ }^{2}$
}

${ }^{1}$ MD Medicine, Senior Resident, Department of Cardiology at IGMC Shimla, Himachal Pradesh, India

${ }^{2}$ MD Radio-diagnosis, Consultant, Radiologist at Sri Ram Hospital, New Shimla, Himachal Pradesh, India

\begin{abstract}
Electrocardiogram (ECG) changes are observed in patients with acute stroke and may create confusion with ECG changes found in cardiac disorders. Various ECG changes are observed in patients with acute stroke including rhythm disturbances (like sinus tachycardia, sinus bradycardia, $A F, V P c, R B B B, L B B B$, etc.) , prolonged QTc interval, ST segment changes, $T$ wave inversion are the most common findings. Atypical flutter in patient with acute stroke is a rare ECG finding observed in our patient.

Keywords: Atypical flutter, ECG, Acute ischemic stroke.
\end{abstract}

\section{INTRODUCTION}

ECG changes are present in $60-90 \%$ of patients with intra-parenchymal or subarachnoid bleed and in about $5-20 \%$ of patients with acute ischemic stroke. ${ }^{1}$ The underlying basis is disordered repolarization process. ${ }^{2}$ The possible mechanism is through disturbances in autonomic regulation and massive stimulation of the sympathetic nervous system. ${ }^{3}$

Sustained sympathetic stimulation results in structural damages to the myocardium, which may be mediated by a sudden increase in intracranial pressure, 4 hypothalamic, ${ }^{5}$ and cardiac nerve stimulation or through an arrhythmogenic center in the insular cortex.6 Moreover, direct damage to the cardiac innervations or imbalance between the left and right sympathetic out flows to the heart, underlying atherosclerotic or hypertensive cardiovascular disease, or asymptomatic/undetectable primary heart disease are among the suggested causes. ${ }^{7,8}$
The ECG abnormalities frequently noted are prolongation of QT interval $45 \%$, ischemic changes $35 \%$ and disturbances in rate and rhythm $25 \%$, which include atrial fibrillation, premature atrial and ventricular complexes, supra-ventricular and ventricular tachycardias (SVT and VT), torasede de pointis or polymorphic ventricular tachycardias. ${ }^{9,10}$

\section{CASE REPORT}

A 24 years old lady presented with history of sudden onset of weakness of left half of body with deviation of angel of mouth to opposite side for last $5 \mathrm{hrs}$. No history of diabetes, hypertension, smoking, pregnancy, hypercoagulable state or trauma.

On physical examination, patient was conscious, cooperative and well oriented to time place and person. Slurring of speech is there. UMN type of left hemiparesis with supranuclear 7th nerve palsy were observed. No sensory neurological deficit was found. Examination of other systems including cardiovascular system were normal. Possibility of 


\section{JMSCR Vol||05||Issue||03||Page 19060-19062||March}

acute stroke was kept and MRI brain was performed.

Routine investigations (including complete haemogram, FBS, Lipid profile, LFT, RFT, examination were done. All of these hematological and biochemical investigations were within normal ranges. While ECG showing Atypical flutter waves.

Electrolytes, Thyroid function tests \&Urine

MRI BRAIN suggestive of Acute Infarct:
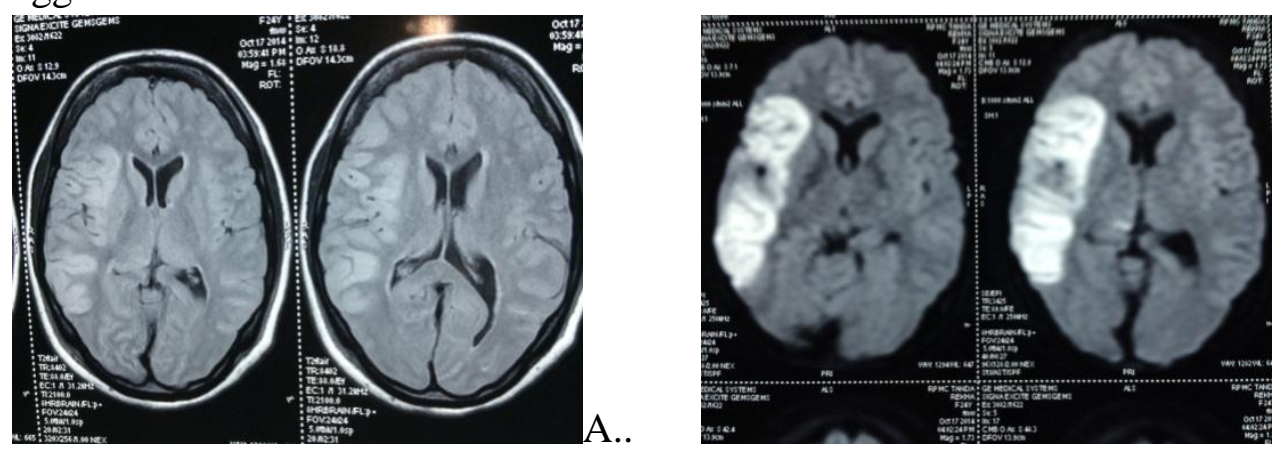

C..

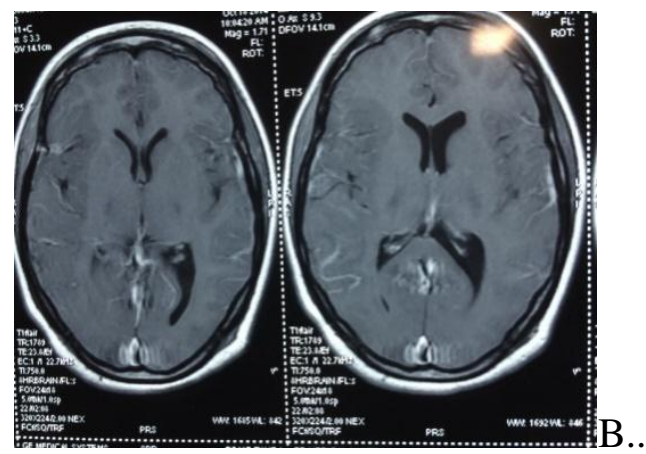

MRI Image: Infarct right fronto-parietal A.T2 FLAIR, B.T1 FLAIR, C.Post contrast enhancement.

ECG showing Atypical Atrial flutter:

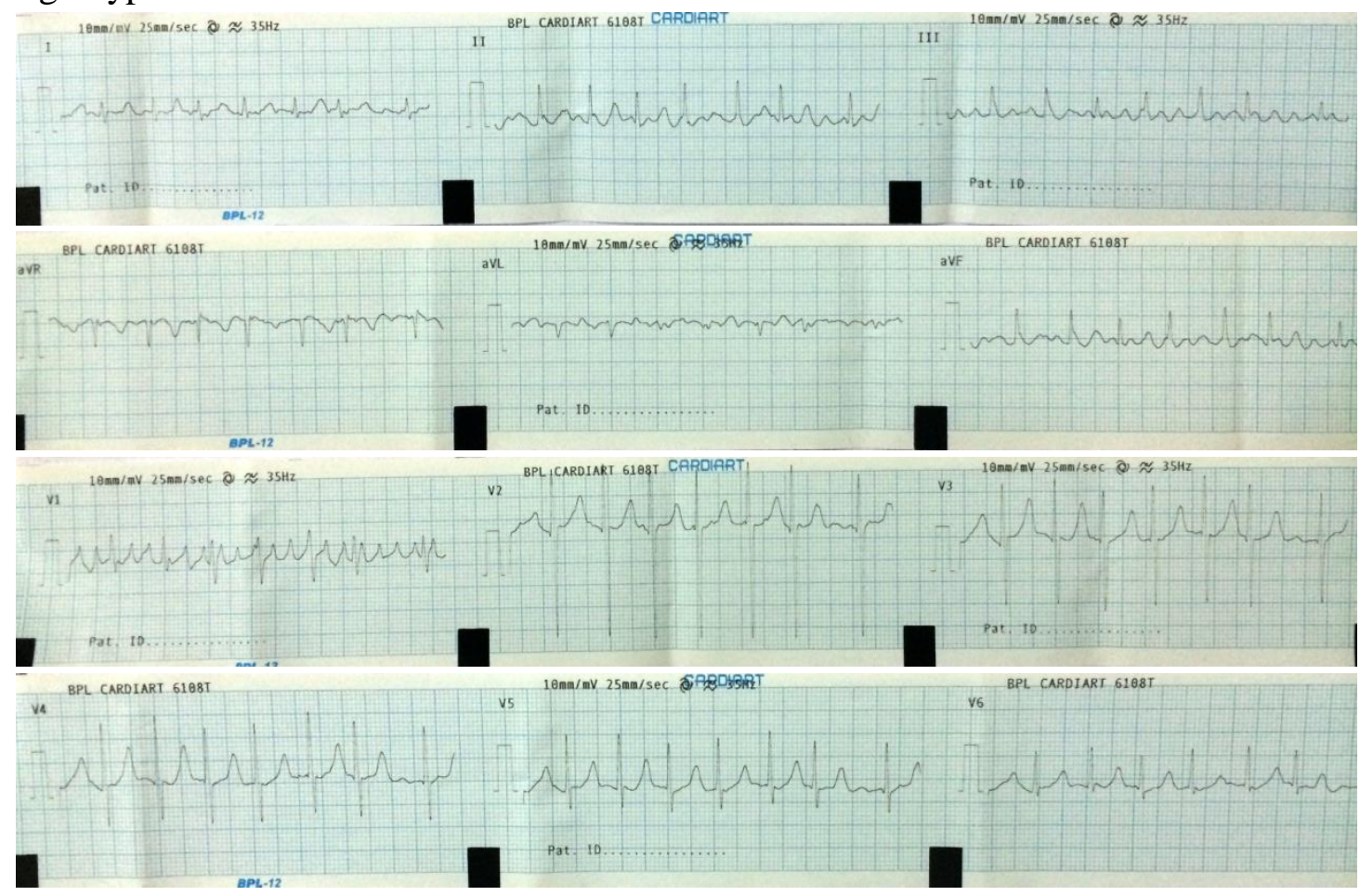

ECG Image: Findings (1.Atypical Flutter waves in leads II, III, Avf\&V1 2.Prolonged QTc interval, QTc $=0.50 \mathrm{sec}$ ) 


\section{CONCLUSION}

Just looking ECG changes we should not directly jump to cardiac diagnosis, we should clinically assess for other etiologies (especially stroke), because management protocol for both etiologies are quite different. Hence may guide us further management. In our patient atypical flutter waves a rare ECG finding may be secondary to ischemic stroke or ischemic stroke may be secondary to atrial flutter. Further studies are required to more precisely clarify the causal connection between these abnormalities and the intracranial lesion.

\section{REFERENCES}

1. Fentz V, Gormsen J. Electrocardiograph patterns in patients with acute cerebrovascular accidents. Circulation. 1962;25:22-8. 4.

2. Oppenheimer SM, Hachinski V. The cardiac consequences of stroke. Neurol Din. 1992;10:167-76. 5.

3. Melville KI, Blum B, Shistr HE. Cardiac ischemic changes and arrhythmias induced by hypothalamic stimulation. Am J Cardiol. 1963;12:78.

4. Shanlin RJ, Sole MJ, Rahimifar M, Tator $\mathrm{CH}$, Factor SM. Increased intracranial pressure elicits hypertension, increased sympathetic activity, electrocardiographic abnormalities and myocardial damage in rats. J Am CollCardiol 1988;12:726-36.

5. Melville KI, Blum B, Shister HE, Silver MD. Cardiac ischemic changes and arrhythmias induced by hypothalamic stimulation. Am J Cardiol 1963;12:781-91.

6. Hirashima Y, Takashima S, Matsumura N, Kurimoto M, Origasa H, Endo S. Right sylvian fissure subarachnoid hemorrhage has electrocardiographic consequences. Stroke 2001;32:2278-81.

7. Natelson BH. Neurocardiology: An interdisciplinary area for the 80s. Arch Neurol 1985;42:178-84.

8. Greenhoot JH, Reichenbach DD. Cardiac injury and subarachnoid hemorrhage: A clinical, pathological and physiological correlation. J Neurosurg 1969;30:521-31.

9. Goldstein DS. The electrocardiogram in stroke: Relationship to pathophysiological type and comparison with prior tracings. Stroke 1979; 10: 253-9.

10. Connor R. Myocardial damage secondary to brain lesions. Am Heart J 1969; 78: 145-8. 